\title{
Modeling and Prediction of Session Throughput of Constant Bit Rate Streams in Wireless Data Networks
}

\author{
Liang Cheng \\ Department of Computer Science and Engineering \\ Lehigh University \\ 19 Memorial Drive West, Bethlehem, PA 18015 \\ cheng@cse.lehigh.edu
}

\author{
Ivan Marsic \\ Center for Advanced Information Processing \\ Rutgers University \\ 96 Frelinghuysen Road, Piscataway, NJ 08854 \\ marsic@caip.rutgers.edu
}

\begin{abstract}
This paper presents an approach to modeling and prediction of session throughput of constant bit rate streams in wireless data networks. A stable traffic generator is used to generate smooth data streams that are transmitted across various types of wireless connections in real-world wireless data networks, including wireless LANs and wireless cellular WANs. The throughput values of the data streaming sessions are recorded. Based on the analysis of statistical properties of the collected data, linear time series analysis is used to model and predict the session throughput. Autoregressive (AR) models are selected from a number of linear time series models since they can be fit to data in a deterministic amount of time. The performance of AR models for prediction is compared to simpler models such as MEAN and Window Mean (WM) models, and our study shows that successful models, such as AR and WM models, have similar performance in predicting the session throughput of wireless data networks. The main contribution of our research is that by statistical study it shows that session throughputs in wireless data networks can be modeled and predicted to a useful degree from past values by using linear time series analysis such as $A R$ and WM models.
\end{abstract}

Keywords-throughput; modeling; prediction; constant-bit-rate; wireless

\section{INTRODUCTION}

\section{A. Wireless Data Networks}

As more and more wireless connections are established in offices and homes, wireless data networks are increasingly involved in our daily life. Various technologies are utilized in wireless data networks, such as Bluetooth [1] in personal area networks (PAN), IEEE 802.11 [2] and HyperLan [3] in wireless local area networks (WLAN), cellular digital packet data (CDPD) [4] in wireless wide area networks (WWAN). In this paper, we focus on the data service in wireless data networks and wireless voice networks [5] providing integrated voice/data services.

\section{B. Motivation}

With the increase of the maximum bandwidth supported by wireless data networks, data applications are proliferating in the wireless communication environment. In general, the session throughout of an application transmitting data across the wireless data networks is apt to volatility because of the dynamics of the wireless links. Therefore it is helpful for the applications to have information of the throughput dynamics so that their performance can be improved by application adaptations.

There are several types of traffic patterns in the data networks, such as constant-bit-rate (CBR) and variable-bit-rate (VBR) traffics. The CBR traffic pattern can be viewed as one of the basic ones since many traffic patterns can be obtained by superposition of various parameterized CBR ones. In this research, our goal is to study the effects of the dynamics of wireless communication links on the session throughput. Since the throughput in wireless networks depends on many factors including the movement of the wireless devices, geographical terrain and obstructions between the transmitter and the receiver, and the wireless networking protocols, we study the feasibility of modeling and prediction of the session throughput of CBR streams in wireless data networks under various conditions, such as in WLAN and WWAN with different physical (PHY) and media-access-control (MAC) layer protocols, and in real-life working environment with walking and driving scenarios when using wireless devices. Thus this paper will focus on the feasibility study. Its applications may also be of interest, but they are not the research foci here.

In addition to allowing applications to adapt to the wireless medium, it is important to have the information about the session throughput for other benefits, by monitoring, modeling and predicting it, in such wireless data networks. For example, planning and managing the wireless data networks can benefit from such information so that troubleshooting and traffic engineering can be performed. How wireless data networks should be designed, tested, and managed is in fact an outstanding issue in the provisioning of wireless data services. By studying session throughput of high data rate CBR sessions across a certain wireless data network, information of dynamics of wireless links can be gathered for service providers' planning decisions.

\section{Related Work}

Previous research work has considered computing resources, including host and network parameters in wired networks [6]. Parameters that have been monitored and 
predicted include: usage of computer resources (CPU, memory), throughput or available throughput of a communication path, latency of a data link, and etc. For example, network weather service (NWS) is a well-known design to provide accurate forecasts of dynamically changing performance characteristics for a distributed set of metacomputing resources [7]. There also exists research on wired networks using statistical models to study the data traffic behavior in both wide-area networks [8][9] and localarea networks [10]. Linear time series models are used in predicting both long-term and short-term Internet conditions and traffic behaviors. The results are used to predict network performance in support of best-effort/real-time services and applications.

There is little existing research on modeling and predicting network parameters in wireless data networks. Noble et al. [11][12] studied the agility and stability of exponentially weighted moving average (EWMA) methods for estimation of available bandwidth in the context of mobile networking. Thus we start the research by studying the feasibility and effectiveness of applying the techniques used in the existing research, such as time-series analysis, to CBR-session throughput modeling and prediction in the wireless data networks.

Our approach is presented and data collection is described

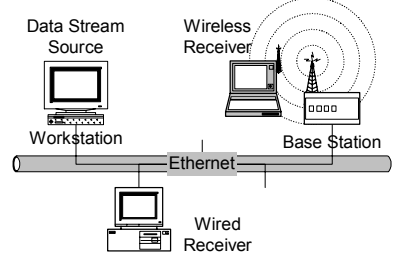

(a) Wireless LAN

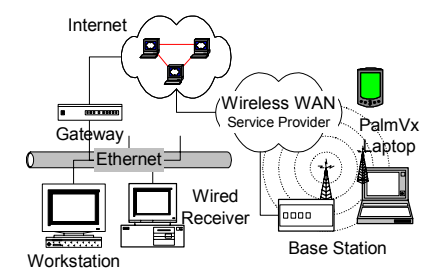

(b) Wireless WAN

Figure 1. Abstract models of experimental networks.

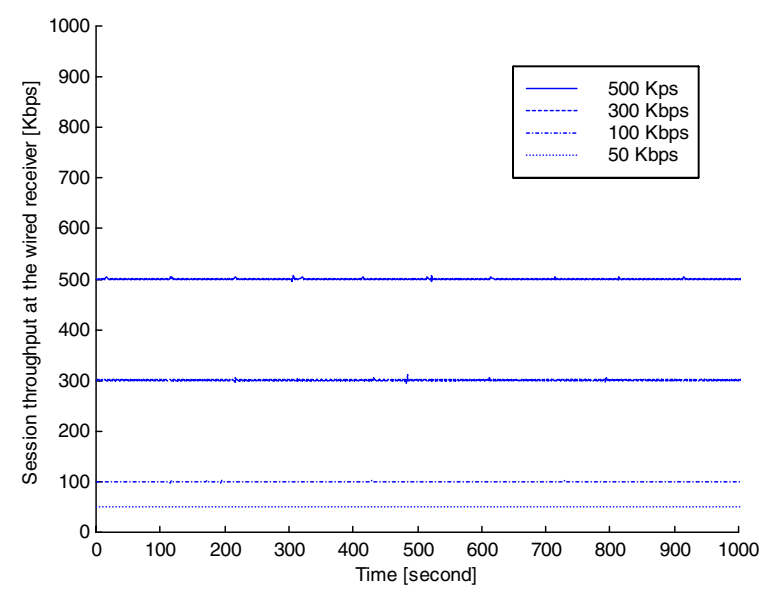

Figure 2. Data streaming traffic received at the wired host in wired LAN. in Section 2. Statistical properties of the collected data sets are studied in Section 3. In Section 4, we compare several modeling techniques and choose appropriate ones for modeling the session throughput of wireless data networks (or wireless session throughput). In Section 5, prediction of wireless session throughput is investigated based on the selected models. Section 6 presents conclusions and the roadmap for future work.

\section{APPROACH}

In general, a modeling and prediction system can be designed in the following steps: $(i)$ performing measurements of network resources; (ii) collecting traces of the measurements; (iii) analyzing and modeling the traces using various, mostly statistical, tools; (iv) evaluating candidate models based on the analysis; and $(v)$ implementing a realtime prediction system based on the appropriate models. We follow these steps in conducting this research.

\section{A. Experiments Description}

Experiments have been designed to measure session throughputs in the wireless data networks. Considering the proliferation of audio and video applications as the data service in data networks, we study the throughput of data streaming sessions across the wireless data networks. Relationship between the wireless session throughput and wireless link bandwidth is discussed.

\section{Experimental Networks}

Fig. 1(a) and Fig. 1(b) illustrate the abstract models of experimental networks in the wireless LAN case and the wireless WAN case. The wireless LANs are campus networks and wireless WANs are commercial networks and both are used simultaneously by multiple users.

The wireless LAN scenarios include Proxim RangeLAN [13] and Sony WirelessLAN [14]. Proxim RangeLAN operates at 2.4 to $2.483 \mathrm{GHz}$ using spread spectrum frequency hopping with media access protocol OpenAir CSMA/CA and delivers data traffic up to $1.6 \mathrm{Mbps}$. Sony WirelessLAN is an IEEE $802.11 \mathrm{~b}$ wireless network operating at $2.4 \mathrm{GHz}$ radio frequency band using direct sequence spread spectrum (DSSS) and delivers up to $11 \mathrm{Mbps}$ which is comparable to wired Ethernet. RTS/CTS is not enabled.

The wireless WAN scenarios include two service providers for CDPD networks. One service provider is OmniSky [15] that provides wireless modems for PalmVx handheld devices. The other is Sierra [16] providing wireless modem cards for mobile laptops.

\section{Data Streaming Source and Sink}

As shown in Fig. 1(a), in the wireless LAN experiments the data stream source or sender is a Sun Sparc10 workstation with Solaris 2.6 platform and the data stream sinks or receivers include a mobile laptop and a wired personal computer, both with Windows NT platform installed. The 


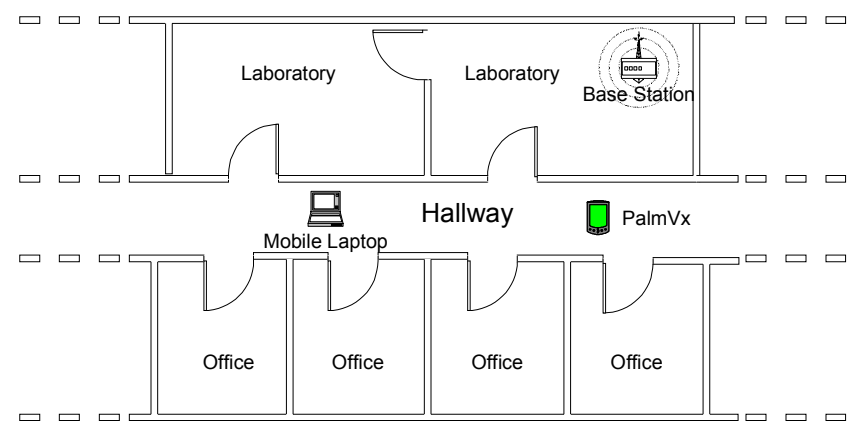

Figure 3. Floor layout in the wireless LAN experiments.

sender sends datagram traffic in a multicast fashion so that both receivers can receive the same data traffic.

A stable traffic generator, which overcomes the randomness caused by the scheduling mechanisms of operating systems and coarse granularity of timers, is used at the source to

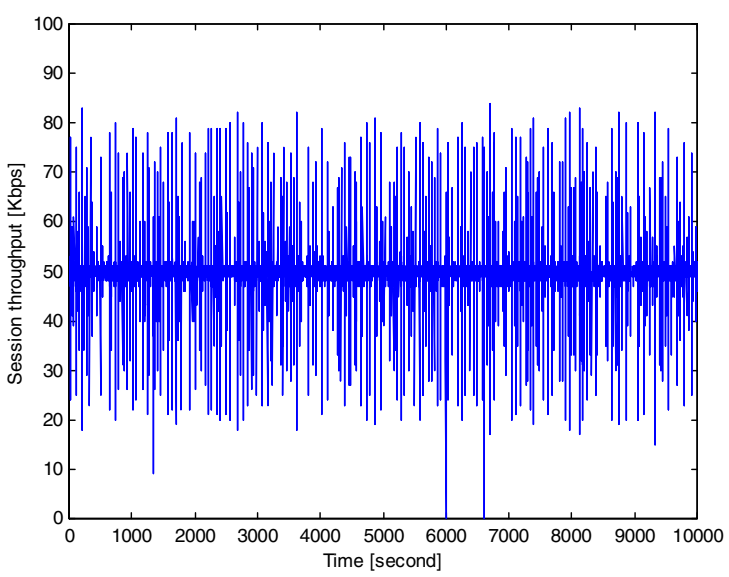

(a) RangeLAN wireless LAN, source rate: $50 \mathrm{Kbps}$

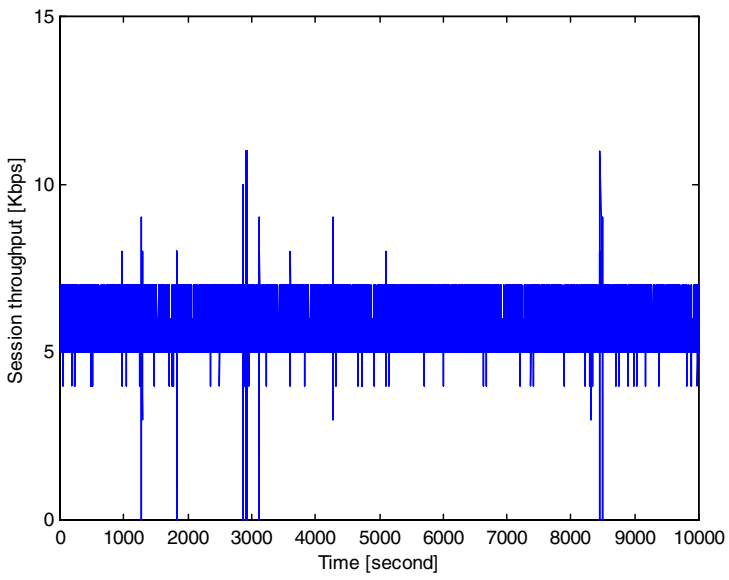

(c) CDPD with Palm, source rate: $6 \mathrm{Kbps}$ generate stable and smooth data streams at fixed rates. Fig. 2 shows the traces of various generated data traffic rates as the wired receiver in the same LAN receives the data stream and records the number of bits received every second, which is defined as the session throughput.

In one wireless WAN scenario in which the PalmVx device is used, the data stream source is the PalmVx and the receiver is the wired personal computer because the PalmVx is not suitable to record large amount of data on the device. In the other wireless WAN case in which mobile laptop is used, the placement of the sender and the receiver is the same as that in the wireless LAN case.

\section{Mobility Scenarios of the Mobile Devices}

Fig. 3 shows the building layout in our experiment. In the wireless LAN case, the mobile laptop travels with varying velocity around the base station (or access point) along the hallway and enters the offices and the labs. (If the velocity is zero, the mobile laptop is stationary.) The maximum distance

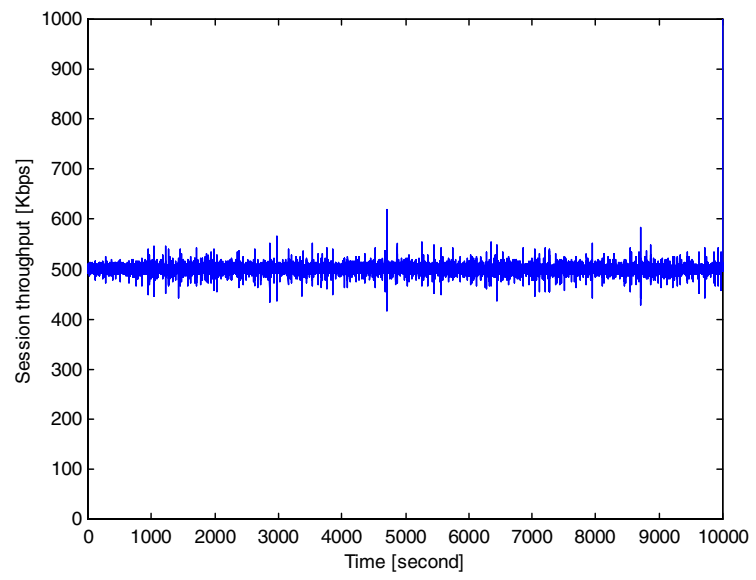

(b) $802.11 \mathrm{~b}$ wireless LAN, source rate: $500 \mathrm{Kbps}$

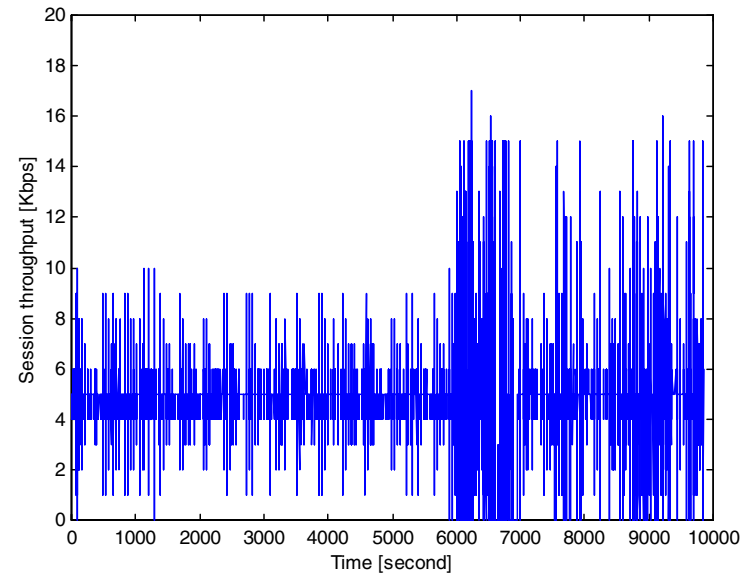

(b) CDPD with laptop, source rate: $5 \mathrm{Kbps}$

Figure 4. Session throughput recorded at the wireless receivers in different groups of experiments. 
between the laptop and the base station reaches 100 feet. Generally there is no line-of-sight communication between the base station and the mobile laptop. In the wireless WAN case, the velocity of the Palm device and the mobile laptop varies from the stationary, walking speed, to driving speed, and the moving paths include paths inside the building in the offices and hallways, and driveways outside the building within a five-mile range.

\section{B. Data Collection}

Based on the above-described experimental scenarios, we categorize the experiments into four groups, namely the RangeLAN, the $802.11 \mathrm{~b}$, the CDPD with PalmVx, and CDPD with laptop. For each group, several data sets of the throughputs recorded at the receivers with different streaming rates are collected. Each data set contains 10,000 values of the throughput, with one value recorded every second. We recorded data sets of throughputs of different sessions with different session sending rates. The collection time of data sets

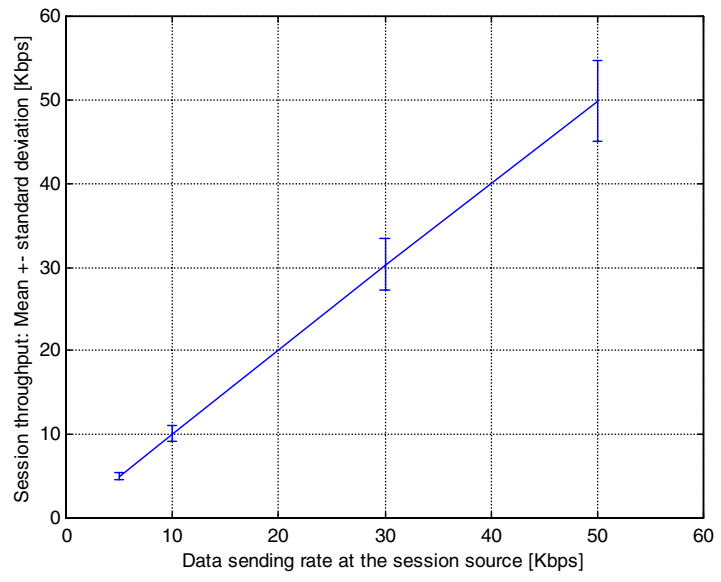

(a) RangeLAN wireless LAN

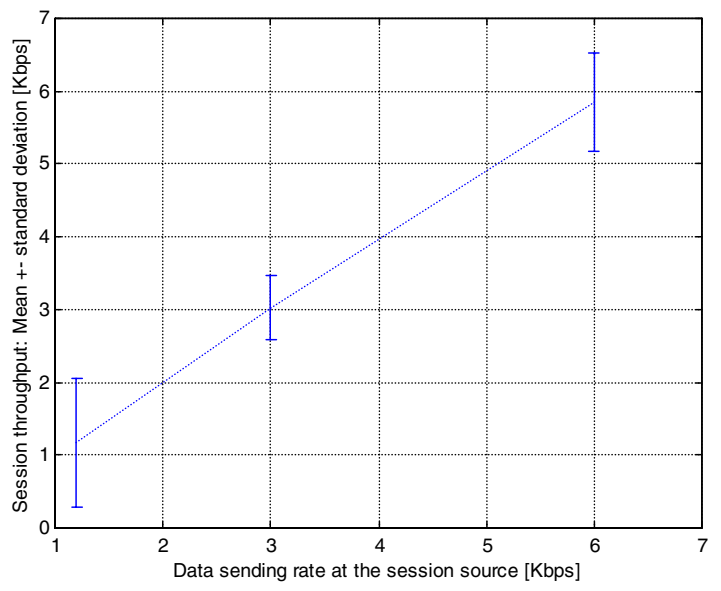

(c) CDPD with Palm is randomly distributed over several weeks. In order to keep the illustrations clear, not all data sets and sample values are plotted in the graphs shown in this paper. We observed that a fraction of all the data sets and sample values in each data set well represents the whole data sets in terms of statistical characteristics. Fig. 4 is a good representative of the general results. Various scales are used for better zoom-in view.

\section{Session Throughput vs. Wireless Link Bandwidth}

A comparison of Fig. 2 and Fig. 4 shows that the data traffic received at the wired host is very different from that received at the mobile hosts. In the wireless LAN case, the difference is solely caused by the dynamics of the wireless link since the mobile and the wired hosts receive the same multicast streaming session and the only difference is the final link type, i.e., wired vs. wireless. Fig. 4 shows that the session throughput changes dynamically in a wide range due to the dynamics of the wireless link.

In the wireless WAN scenarios the receiving host is not in

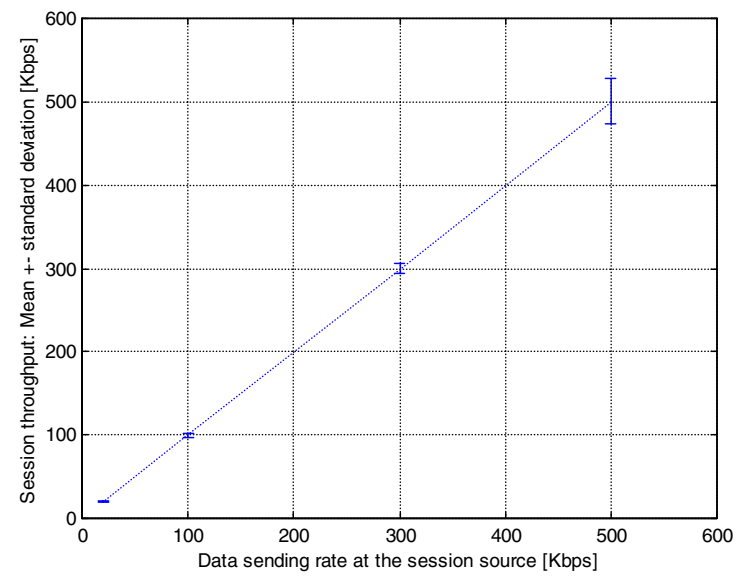

(b) $802.11 \mathrm{~b}$ wireless LAN

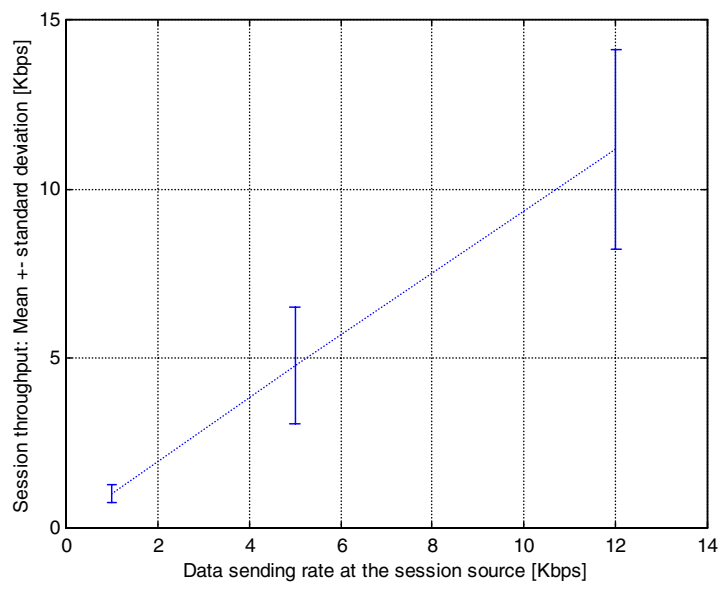

(d) CDPD with laptop

Figure 5. Mean bandwidth $+/$ - standard deviation of the session throughput in different groups of experiments. 
the same subnet as the base station because we do not have control over the commercial setup. However, we believe that the dynamics of the throughput is still mainly caused by the wireless links in wireless WANs. In our experiments, the difference between the nominal source rate and the throughput recorded at the receiver is determined by the packet delay, loss and retransmission. The packet loss and packet retransmission can be viewed as special cases of the packet delay. According to [17], the mean value of packet delay in wired networks is much smaller than that in wireless networks. Therefore it is assumed that the measured throughput reflects the wireless link bandwidth even in the wireless WANs.

\section{StATISTICAL PROPERTIES}

\section{A. Mean and Standard Deviation}

Summarizing each data set in terms of its mean, standard deviation, and maximum and minimum values illustrates the extent to which the session throughput varies. Fig. 5 shows the mean value and the plus and minus one standard deviation points for different data sets. It reveals that the standard

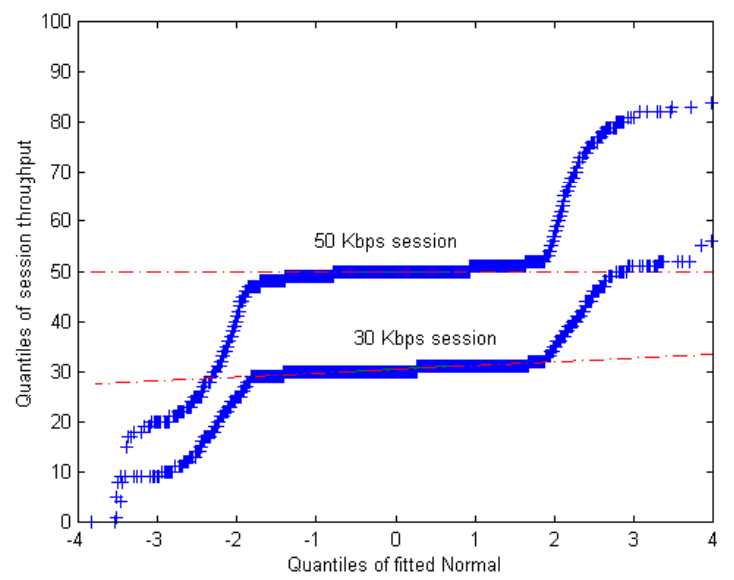

(a) RangeLAN wireless LAN

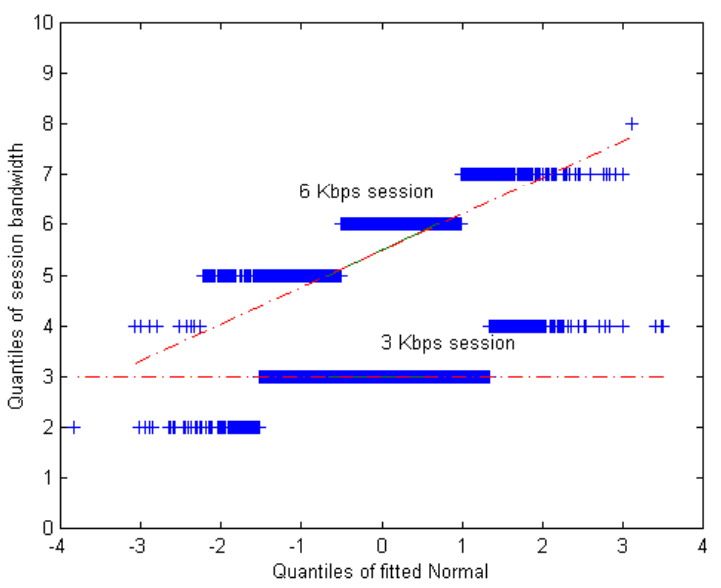

(c) CDPD with Palm

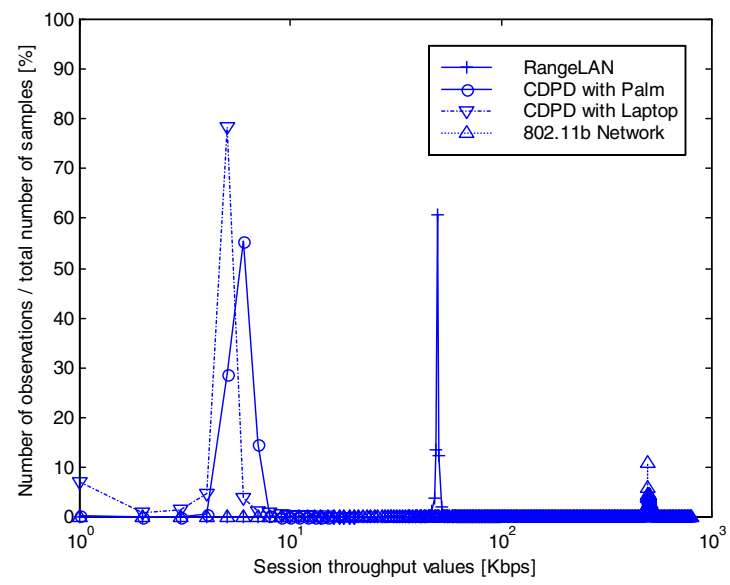

Figure 6. Histogram of the data sets shown in Figure 4.

deviation, in absolute terms, grows with the increasing mean throughput. However, the trend does not exist in relative terms if we express it in terms of the coefficient-of-variation (COV)

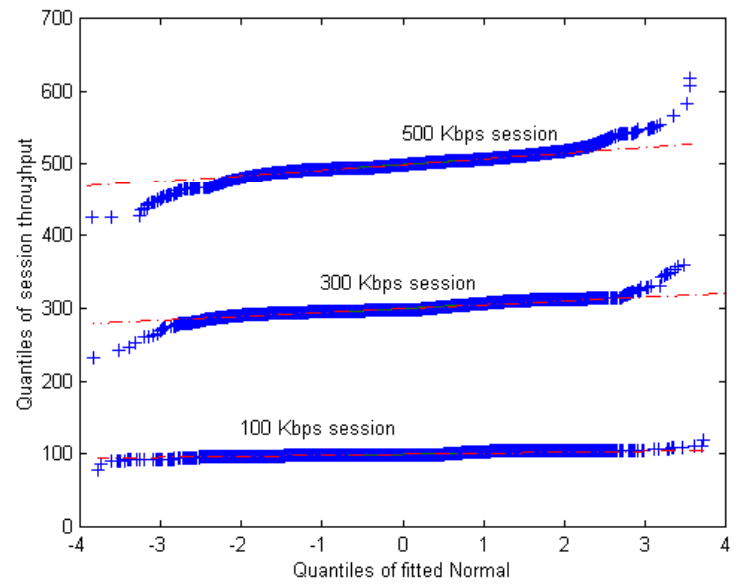

(b) $802.11 \mathrm{~b}$ wireless LAN

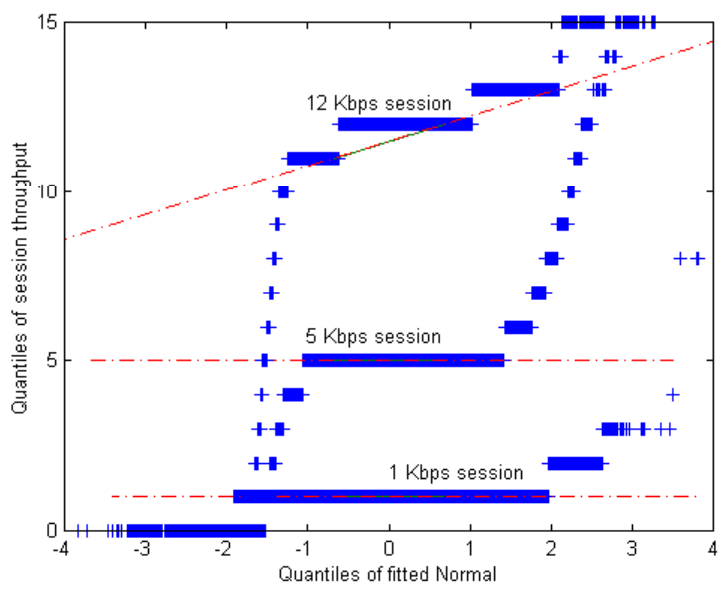

(d) CDPD with laptop

Figure 7. Quantile-quantile plots: session throughput vs. normal distribution. 


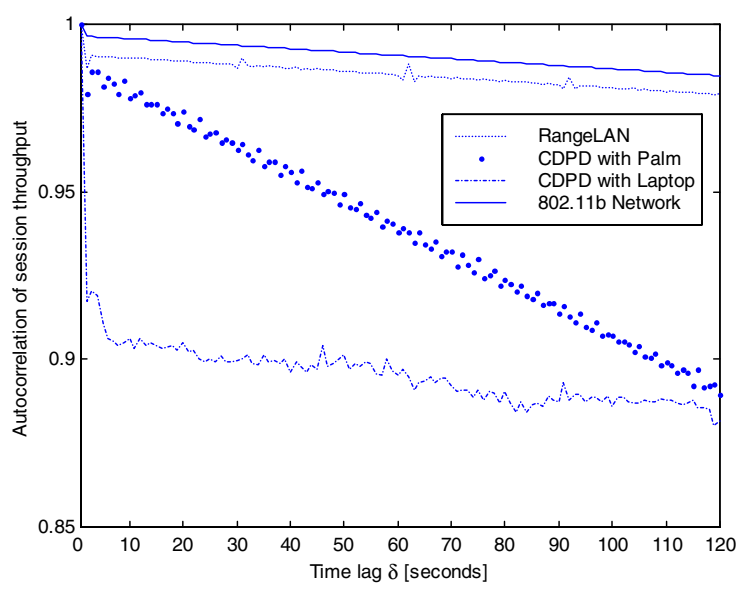

Figure 8. Autocorrelation function of data sets in Figure 4.

as the standard deviation divided by the mean. We also observed that the mean values in all the data sets do not vary with time if statistically significant amount of consecutive samples are considered, e.g., 1,000.

\section{B. Distribution}

Fig. 6 plots the histograms of data sets in Fig. 4, normalized as the percentage of the number of occurrences over the total number of samples. It shows that the distribution has a unimodal shape. We observed that all other data sets have the unimodal distribution pattern. If we treat each data set as a realization of an independent, identically distributed (IID) stochastic process, then the histogram obtained from a large number of data closely approximates its probability distribution function ( $\mathrm{pdf}$ ), which can completely describe the IID stochastic process.

We fit a normal distribution to each data set. Fig. 7 shows quantile-quantile plots for the normal distribution fitted to the several data sets in each group. According to the property of quantile-quantile plots, the plot will be linear if the distribution fits the data. Fig. 7 implies that the normal distribution does not fit the distributions of the data sets well.

\section{Time Series Analysis}

We examined the autocorrelation function of each data set. The autocorrelation quantifies how well a throughput value at time $t$ is linearly correlated with its corresponding throughput value at time $t+\Delta$, which in turn shows how well the value at time $t$ predicts the value at time $t+\Delta$. The value of autocorrelation function ranges between -1 and 1 . The closer the value to 1 , the better linear correlation of the value at time $t$ and time $t+\Delta$. The time series analysis shows that the past throughput value has a strong influence on the future throughput value. For example, Fig. 8 shows the autocorrelation function to a lag of up to 120 seconds (2 minutes) for the data set in Fig. 4. Note that in all scenarios, including the WLAN and WWAN networks, the values of session throughput are strongly correlated.

We found that the above statistical properties are present in the whole data sets, irrespective of the velocity of the mobile receivers. This implies that session throughput prediction based on past values is feasible using linear time series models.

\section{MODELING}

There are a number of models for linear time series analysis, such as autoregressive (AR), moving average (MA), autoregressive moving average (ARMA), autoregressive integrated moving average (ARIMA), and autoregressive fractionally integrated moving average (ARFIMA) models [18]. Mostly ARIMA and ARFIMA are used in the nonstationary time series analysis. Due to resource constraints and the computational complexity of ARIMA and ARFIMA models, we only consider AR, MA, and ARMA models here.

\section{A. ARMA Models}

If a data set with time series value $\left\{X_{t}\right\}$ can be modeled by an ARMA model, then it can be described as follows:

$\left\{X_{t}\right\}$ is an $\operatorname{ARMA}(p, q)$ process if $\left\{X_{t}\right\}$ is stationary and if for every $t$

$$
X_{t}-\phi_{1} X_{t-1}-\ldots-\phi_{p} X_{t-p}=Z_{t}+\theta_{1} Z_{t-1}+\ldots+\theta_{q} Z_{t-q}
$$

where $\left\{Z_{t}\right\}$ is a white noise sequence $\mathrm{WN}\left(0, \sigma^{2}\right)$. It is convenient to use a concise form as

$$
\phi(B) X_{t}=\theta(B) Z_{t}
$$

where $\phi(\cdot)$ and $\theta(\cdot)$ are the $p^{\text {th }}$ and $q^{\text {th }}$ degree polynomials as

$$
\begin{aligned}
& \phi(x)=1-\phi_{1} x-\ldots-\phi_{p} x^{p} \\
& \theta(x)=1+\theta_{1} x+\ldots+\theta_{q} x^{q}
\end{aligned}
$$

and $B$ is the backward shift operator $\left(B^{j} X_{t}=X_{t-j}, B^{j} Z_{t}=Z_{t-j}\right.$, $j=0, \pm 1, \pm 2 \ldots)$. The time series model is said to be an autoregressive model of order $p$ or $\operatorname{AR}(p)$ if $\theta(\cdot) \equiv 1$ and a moving average model of order $q$ or $\operatorname{MA}(q)$ if $\phi(\cdot) \equiv 1$.

\section{B. AR Models}

Note that MA and ARMA models are a much more difficult proposition for a system designer since modeling time series data by them takes a non-deterministic amount of time. Instead of a linear system, fitting a MA or ARMA model present us with a quadratic system. Thus AR models are highly desirable since they can be fit to data in a deterministic amount of time. For example, in an AR model with $p$-order using the YuleWalker technique [18], the autocorrelation function is computed to a maximum lag $p$ and then a $p$-wide Toeplitz system of linear equations is solved. Even for relatively large values of $p$, this can be done almost instantaneously. The evaluation results presented below demonstrate that $A R$ models are sufficiently accurate for modeling the session throughput of wireless data networks. We also compare AR 
models with simple models such as MEAN and windowed mean (WM) [7].

\section{Simple Models}

The MEAN model has $X_{t}=\mu$, so the future values of the time sequence are predicted to be the mean. The WM model simply predicts the next sequence value to be the average of the previous $p$ values, a simple windowed mean. Note that WM subsumes an even simpler model: LAST model as "predict that the next value will be the same as the last value", i.e., $p=1$.

\section{Exponential Model}

Variations of exponential model have been used and studied in [7] for the estimation/prediction of network parameters as well as in TCP for round-trip time estimation [19]. It can be expressed as follows:

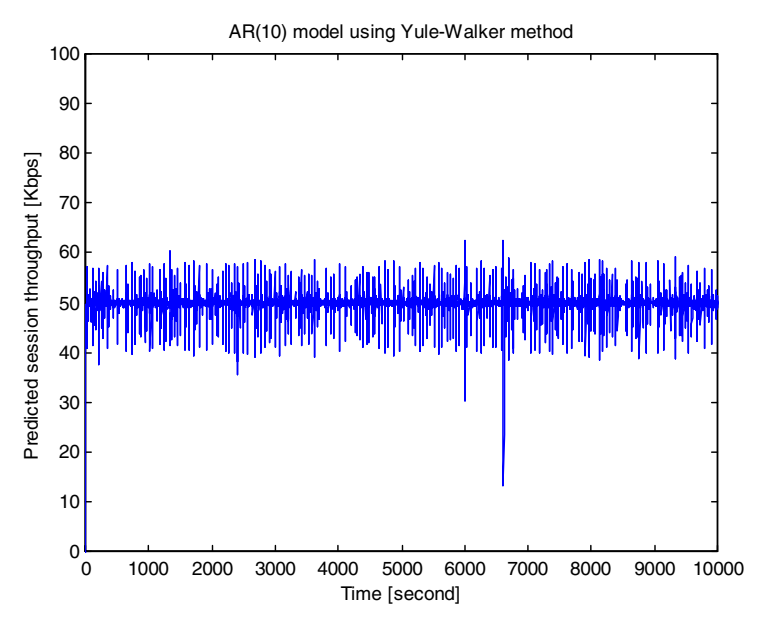

(a) Predicted session throughput (1-second prediction)

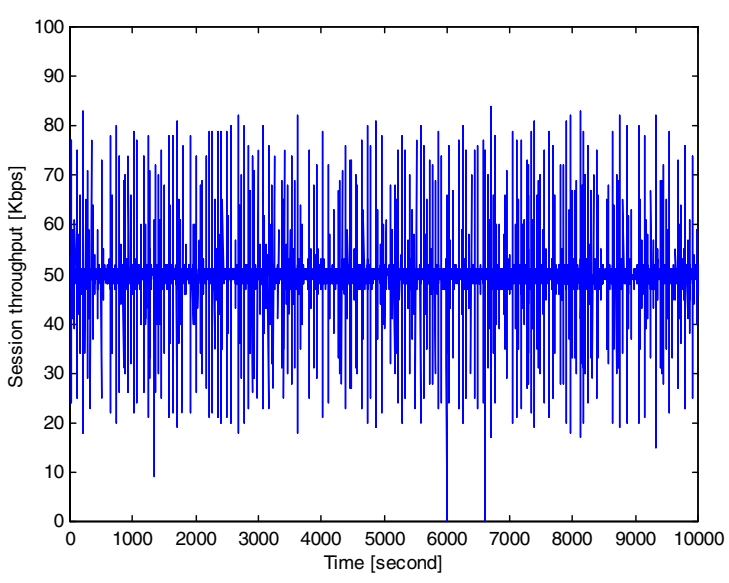

(b) Observed session throughput

Figure 9. Predicted vs. observed session throughput in the $50 \mathrm{Kbps}$ case in wireless LAN shown in Figure 4a.

$$
x_{t}^{p}=\alpha x_{t-1}+(1-\alpha) x_{t-1}^{p}
$$

where $x^{p}{ }_{t}$ is the predicted value of $x$ at time instance $t$, and $x_{t-1}$ is the actual value at time instance $t-1$.

This is a special form of AR model for prediction. From Eq. (5),

$$
x_{t-1}^{p}=\alpha x_{t-2}+(1-\alpha) x_{t-2}^{p}
$$

Substitute Eq. (6) to Eq. (5):

$$
x_{t}^{p}=\alpha x_{t-1}+\alpha(1-\alpha) x_{t-2}+(1-\alpha)^{2} x_{t-2}^{p}
$$

Perform similar substitutions recursively, then:

$$
x_{t}^{p}=\alpha x_{t-1}+\ldots+\alpha(1-\alpha)^{j-1} x_{t-j}+(1-\alpha)^{j+1} x_{t-j-1}^{p}
$$

If the last item becomes negligible once $j$ becomes large enough, say, $N$, since $\alpha<1$, then:

$$
x_{t}^{p}=\alpha x_{t-1}+\ldots+\alpha(1-\alpha)^{N-1} x_{t-N}
$$

\section{EVALUATION}

We collect additional data sets other than those collected in Section 2 and use all of them for evaluating different models. One-step-ahead and multi-step-ahead predictions are used to evaluate the correctness of different models, such as $\operatorname{AR}(p)$, MEAN, WM $(w)$ models. In the case of sampling frequency equal 1 second, $m$-step-prediction means predicting the throughput value at time instance that is $m$ seconds ahead of current time instance. The performance index is the mean square fit, which is expressed as follows.

Suppose $X$ is the vector of the observed values, and the $X^{\text {pred }}$ is the vector of the predicted values. Then the mean square fit is:

$$
\left\|X-X^{\text {pred }}\right\| / \operatorname{sqrt}(\text { length }(X))
$$

where $\|\cdot\|$ is the norm operator.

Fig. 9 compares the one-second prediction value of session

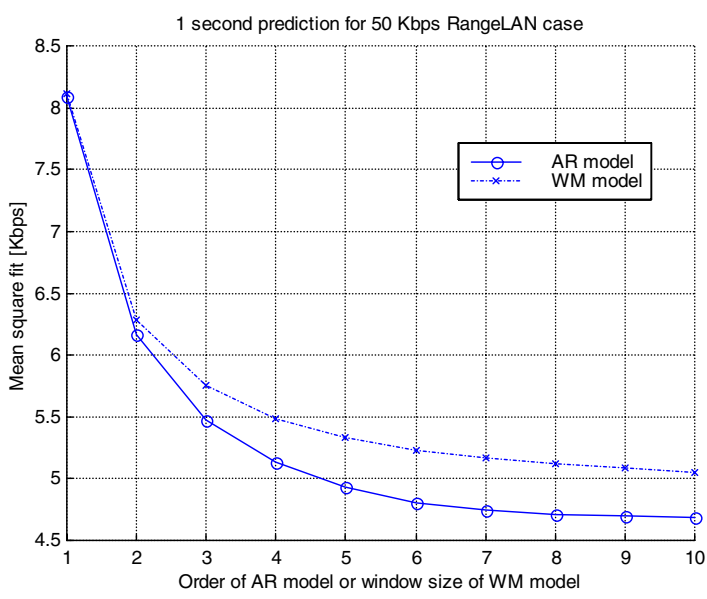

Figure 10. Mean square fit of different models (1 second prediction). 
throughput by AR(10) model using the Yule-Walker method to the observed session throughput in the $50 \mathrm{Kbps}$ case of RangeLAN shown as Fig. 4(a). Fig. 10 shows the mean square fits of $\operatorname{AR}(p)$ models with different orders of $p$ and $\operatorname{WM}(w)$ models with different window size $w$, when predicting session throughput in the $50 \mathrm{Kbps}$ case of RangeLAN. The mean square fit of the MEAN model in this case is 4.7979. Note that unlike the WM model, the MEAN model has to use an infinite buffer to store history data or an unlimited data size to store the sum and the number of the history data as the time increases, which is infeasible in practical modeling and prediction systems.

Using the same evaluation methods described above, we studied the prediction performance of AR model and WM model in the other three groups of data sets, i.e., the cases of 802.11b networks and the CDPD networks with Palm and Laptop. The results show that the prediction errors by all models decrease as the order of the models increase. Moreover, in the cases of WLAN and CDPD with Palm, when the models' order is high enough, e.g., 10, different models

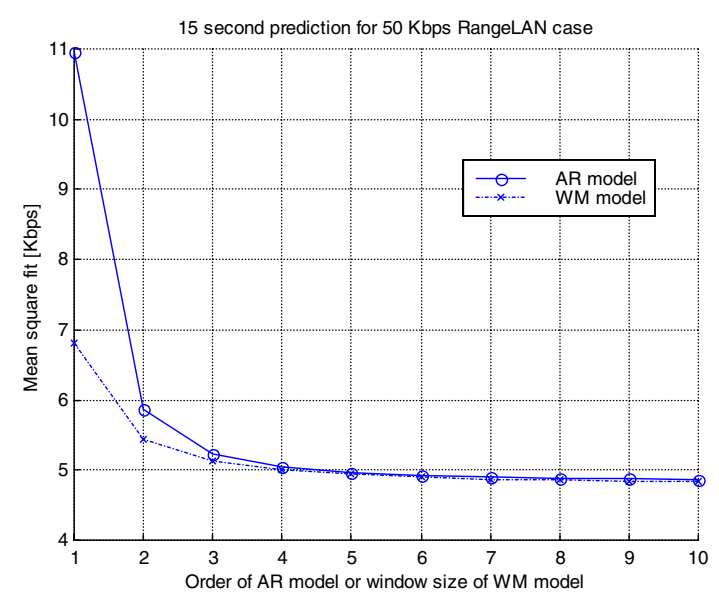

(a) Mean square fit of different models (15-second prediction)

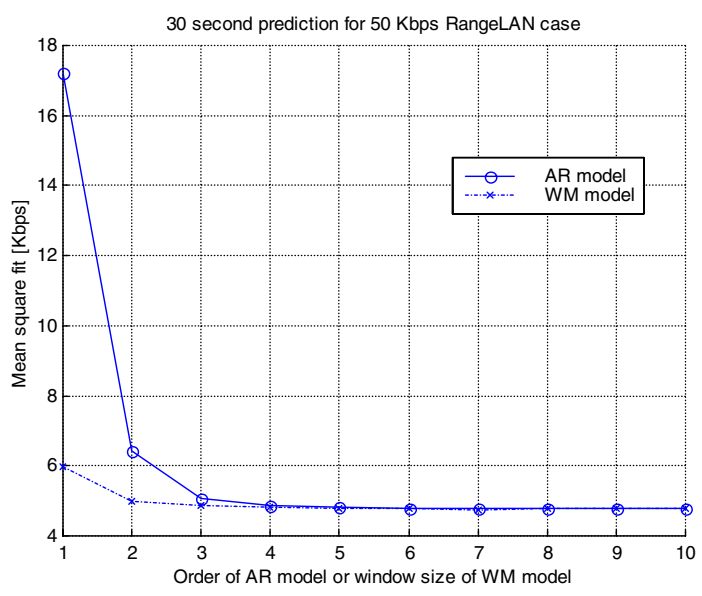

(b) Mean square fit of different models (30-second prediction)

Figure 11. Mean square fit of different models in multi-step-prediction. show similar prediction accuracy and the nominal prediction errors are in the range of $10 \%$. In the case of CDPD with laptop, even though the nominal prediction errors of AR and WM models with high orders are in the range of $30 \%$, the absolute errors are within $1.6 \mathrm{Kbps}$ in the case of a $5 \mathrm{Kbps}$ session. This is due to the bad network connection during the data session since the mobile device was in the blind spots of the CDPD service areas so that there was no service for a large amount of time in such experiments.

Fig. 11 shows the performance of AR model and WM model in multi-step-prediction cases for a $50 \mathrm{Kbps}$ session in RangeLAN. The figures show the 15 seconds prediction and the 30 seconds prediction, respectively. According to Fig. 10 and Fig. 11, we observe that the performances of the AR model and WM model are almost the same when their orders are high enough, e.g., 5. This result remains true in all experimental scenarios.

\section{CONCLUSIONS}

\section{A. Distribution of Wireless Session Throughput}

In this paper, we treat a sequence of values of the session throughput as a realization of an independent, identically distributed (IID) stochastic process. However, the normal distribution does not fit the distribution of the values well. Also, our study of quantile-quantile plots shows that the prediction errors are not IID normal either.

\section{B. Linear Time Series Analysis}

Linear time series analysis is feasible for modeling and prediction of the time sequence values of session throughput of CBR streams in wireless data networks. Specifically, $(i)$ session throughput is consistently predictable because of the strong correlation between the past values and the present/future values; and (ii) successful models, such as autoregressive (AR) and window mean (WM) have similar performance in predicting the session throughput of wireless data networks.

Our future work includes applying these research results to more network scenarios with various traffic patterns and different wireless network performance indices, such as packet loss rate and link delay, and deploying the prediction tools into real world applications.

\section{ACKNOWLEDGMENTS}

This research is supported by NSF KDI Contract No. IIS98-72995, US Army CECOM Contract No. DAAB07-00-DG505, Cisco Systems, Inc., and by the Rutgers Center for Advanced Information Processing (CAIP).

\section{REFERENCES}

[1] The Official Bluetooth SIG Website, http://www.bluetooth.com/

[2] B. O'Hara and A. Petrick, The IEEE 802.11 Handbook: A Designer's Companion, IEEE Press, 1999 
[3] R. LaMaire, A. Krishna, P. Bhagwat, J. Panian, "Wireless LANs and mobile networking: standards and future directions," IEEE Communications Magazine, Vol. 34, No. 8, pp. 86-94, August 1996

[4] P. Sinha, N. Venkitaraman, R. Sivakumar, V. Bharghavan, "WTCP: A reliable transport protocol for wireless wide-area networks," Proc. ACM MobiCom'99, pp.231-241, Seattle, WA, August 1999

[5] Richard A. Schaffer. "High-level computing (mobile technology)," Forbes, vol. 156, no. 8, pp. 116, October 9, 1995

[6] P. Dinda, D. O'Hallaron, "Host load prediction using linear models," Cluster Computing, Vol. 3, No. 4, pp. 265-280, 2000

[7] R. Wolski, "Dynamically forecasting network performance using the Network Weather Service," UCSD Technical Report TR-CS96-494, Computer Science and Engineering Department, University of California, San Diego, January 1998. Online at: http://wwwcse.ucsd.edu/users/rich/publications.html

[8] H. Balakrishnan, M. Stemm, S. Seshan, and R.H. Katz, "Analyzing stability in wide-area network performance," Proceedings of the ACM SIGMETRICS'97, pp. 2-12, 1997

[9] V. Paxson and S. Floyd, "Wide-area traffic: the failure of Poisson modeling," IEEE/ACM Transactions on Networking, Vol. 3, No. 3, pp. 226-244, June 1995

[10] H. J. Fowler and W. E. Leland, "Local area network traffic characteristics, with implications for broadband network congestion management," IEEE JSAC, Vol. 9, No. 7, pp. 1139-1149, September 1991

[11] M. Kim and B. D. Noble, "Mobile network estimation," Proceedings of Seventh ACM Conference on Mobile Computing and Networking (MobiCom'2001), pp. 298-309, Rome, Italy, July 2001

[12] B. D. Noble, L. Li, and A. Prakash, "The case for better throughput estimation," in Proceedings of the 7th Workshop on Hot Topics in Operating Systems, pp. 70-75, Rio Rico, AZ, March 29-30, 1999

[13] Proxim Inc., http://www.proxim.com/

[14] Sony Computing Products, http://www.ita.sel.sony.com /products/pc/notebook/accessories/wlan.html

[15] OmniSky Corp., http://www.omnisky.com

[16] Sierra Wireless Inc., http://www.sierrawireless.com/

[17] L. Cheng and I. Marsic, "Fuzzy reasoning for wireless awareness," International Journal of Wireless Information Networks, Vol. .8, No.1, pp. 15-26, January 2001

[18] G.E.P. Box, G.M. Jenkins, and G. Reinsel, Time Series Analysis: Forecasting and Control, $3^{\text {rd }}$ ed., Prentice Hall, 1994

[19] L. Peterson and B. Davie, Computer Networks: A Systems Approach, $2^{\text {nd }}$ ed., Morgan-Kaufmann Publisher, San Francisco, CA, 1999

[20] P. Dinda, "The statistical properties of host load," Scientific Programming, Vol. 7, No. 3-4, pp. 211-229, Fall 1999 